\title{
Conditions for optimal construction of two-qubit non-local gates
}

\author{
Yong-Sheng Zhang*, Ming-Yong Ye, and Guang-Can Guo ${ }^{\dagger}$ \\ Laboratory of Quantum Information, University of Science and Technology of \\ China, Hefei 230026, People's Republic of China
}

\begin{abstract}
Optimal implementation of quantum gates is crucial for designing a quantum computer. The necessary condition for optimal construction of a two-qubit unitary operation is obtained. It can be proved that the $\mathrm{B}$ gate is the unique gate that can construct a two-qubit universal circuit with only two applications, i.e. this condition is also sufficient in the case of two applications of the elementary two-qubit gate. It is also shown that one half of perfect entanglers can not simulate an arbitrary two-qubit gate with only 3 applications.
\end{abstract}

PACS number(s): 03.67.Lx, 03.67.-a

Quantum computation can be described by unitary matrices. In order to effect a quantum computation on a quantum computer, one must decompose the corresponding unitary matrix into a quantum circuit which consists of elementary quantum gates [1]. It has been shown that any interaction that can create entanglement between any pair of qudits (qubits) is universal for quantum computation together with one-qudit (qubit) gates [2-4]. Many efforts are devoted to the optimal construction of explicit quantum circuits for arbitrary unitary operation. The current standard paradigm is based on a combination of quantum Controlled-NOT (CNOT) gates between pairs of qubits and single-qubit gates [5]. Recent work shows that the CNOT gate is also one of the most efficient quantum gates known, in that just three applications supplemented with local gates can implement any arbitrary two-qubit operation $[6]$.

However, a practical Hamiltonian can not necessarily be efficient to construct the CNOT gate. It is possible to construct different elementary gates that depend on the given Hamiltonian. Questions are raised that are there any gates which have more efficiency than CNOT, or what is the optimal construction of a circuit if the elementary two-qubit gate is not a standard CNOT gate? J. Zhang et al. have proposed optimal construction with B gate and Controlled-Unitary (Controlled-U) gate respectively $[7,8]$. Bremner et al. have given an operable construction with an arbitrary two-qubit elementary gate, though this construction is not always optimal [9].

In this paper, the necessary condition of optimal construction for a two-qubit gate with a general elementary entangling gate is proposed. Nontrivial results in the special cases of two or three applications of the elementary gate are also proposed.

It is known that an arbitrary two-qubit unitary transformation $U$ can be decomposed into this form $[10,11]$.

$$
\begin{aligned}
U & =\left(A_{1} \otimes B_{1}\right) e^{i H_{U}}\left(A_{2} \otimes B_{2}\right), \\
H_{U} & =c_{1} \sigma_{x} \otimes \sigma_{x}+c_{2} \sigma_{y} \otimes \sigma_{y}+c_{3} \sigma_{z} \otimes \sigma_{z} .
\end{aligned}
$$

where $\sigma_{x}, \sigma_{y}, \sigma_{z}$ are the Pauli matrices, $A_{j}, B_{j}$ are single qubit gates, and $\frac{\pi}{4} \geq c_{1} \geq c_{2} \geq\left|c_{3}\right|$. The non-local content $\phi(U)=\lambda\left(H_{U}\right)$ are

$$
\begin{aligned}
\lambda_{1} & =c_{1}+c_{2}-c_{3}, \\
\lambda_{2} & =c_{1}-c_{2}+c_{3}, \\
\lambda_{3} & =-c_{1}+c_{2}+c_{3}, \\
\lambda_{4} & =-c_{1}-c_{2}-c_{3},
\end{aligned}
$$

where $\lambda(A)$ denotes the vector whose entries are eigenvalues of Hermitian matrix $A$, arranged into non-increasing order. In the following, we will denote $e^{i H_{U}}$ as $U_{d}\left(c_{1}, c_{2}, c_{3}\right)$.

Our first result is based on the lemmas which were proposed by Childs et al. [13] and the following theorem of $\mathrm{K}$. Fan [12].

\footnotetext{
*Electronic address: yshzhang@ustc.edu.cn

${ }^{\dagger}$ Electronic address: gcguo@ustc.edu.cn
} 
Lemma 1. Let $H, K$ be Hermitian matrices. Then

$$
\lambda(H+K) \prec \lambda(H)+\lambda(K),
$$

where the majorization relation whose notation is $\prec$ will be described as follows. Suppose $x=\left(x_{1}, \cdots, x_{D}\right)$ and $y=\left(y_{1}, \cdots, y_{D}\right)$ are two D-dimensional real vectors. $x$ is majorized by $y$, written $x \prec y$, if

$$
\sum_{j=1}^{k} x_{j}^{\downarrow} \leq \sum_{j=1}^{k} y_{j}^{\downarrow}
$$

for $k=1, \cdots, D-1$, and the inequality holds with equality where $k=D$. Here $\downarrow$ denotes the components of a vector rearranged into non-increasing order.

Combining Lemma 4, Lemma 6 of Ref. [13], and the above Lemma 1, it is not difficult to obtained the following theorem.

Theorem 2. Let $U_{1}, U_{2}$ be two-qubit unitary operations, then

$$
\phi\left(U_{1} U_{2}\right) \prec \phi\left(U_{1}\right)+\phi\left(U_{2}\right) .
$$

Proof. We denote $U_{0}=U_{1} U_{2}$. According to Lemma 4 of Ref. [13], there exist $H_{j}$ 's $(j=0,1,2)$ that satisfy

$$
U_{j} \widetilde{U}_{j}=e^{2 i H_{j}} \text { and } \lambda\left(H_{j}\right)=\phi\left(U_{j}\right) .
$$

According to Lemma 6 of Ref. [13], there exist $K_{1}$, and $K_{2}$ that satisfy

$$
\lambda\left(U_{0} \widetilde{U}_{0}\right)=\lambda\left(e^{2 i\left(K_{1}+K_{2}\right)}\right),
$$

where $\lambda\left(K_{1}\right)=\lambda\left(H_{1}\right)$ and $\lambda\left(K_{2}\right)=\lambda\left(H_{2}\right)$. According to Lemma 4 of Ref. [13], there must be

$$
\lambda\left(K_{1}+K_{2}\right)=\phi\left(U_{0}\right)+\pi \vec{m},
$$

where $\vec{m}$ is an integer vector. With the above K. Fan's Lemma, it can be obtained that

$$
\begin{aligned}
\phi\left(U_{1}\right)+\phi\left(U_{2}\right) & =\lambda\left(K_{1}\right)+\lambda\left(K_{2}\right) \\
& \succ \lambda\left(K_{1}+K_{2}\right) \\
& =\phi\left(U_{0}\right)+\pi \vec{m} .
\end{aligned}
$$

To satisfy this relation, there must be (Here $\vec{m}$ is written in non-increasing order but $\phi\left(U_{0}\right)$ is not necessarily written in this order.)

$$
\vec{m}=(0,0,0,0) \text { or } \vec{m}=(1,0,0,-1) .
$$

It is not difficult to obtained that

$$
\phi^{\prime}\left(U_{0}\right)+\pi(1,0,0,-1) \succ \phi\left(U_{0}\right),
$$

where $\phi^{\prime}\left(U_{0}\right)$ is any permutation of $\phi\left(U_{0}\right)$. So

$$
\phi\left(U_{1}\right)+\phi\left(U_{2}\right) \succ \phi\left(U_{1} U_{2}\right) .
$$

Though we can get conditions for gate simulation directly in the same way of Hamiltonian simulation [13] with theorem 2, the next lemma gives more strict constraints than Hamiltonian simulation.

Lemma 3. Two consecutive Swap-class gates are equivalent to local unitary operation.

Proof. It is known any Swap-class gate is equivalent to $U_{d}\left(\frac{\pi}{4}, \frac{\pi}{4}, \frac{\pi}{4}\right)$. So

$$
\begin{aligned}
& \left(A_{1} \otimes B_{1}\right) U_{d}\left(\frac{\pi}{4}, \frac{\pi}{4}, \frac{\pi}{4}\right)\left(U_{A} \otimes U_{B}\right) U_{d}\left(\frac{\pi}{4}, \frac{\pi}{4}, \frac{\pi}{4}\right)\left(A_{2} \otimes B_{2}\right) \\
= & \left(A_{1}^{\prime} \otimes B_{1}^{\prime}\right)(\text { Swap })\left(U_{A}^{\prime} \otimes U_{B}^{\prime}\right)(\text { Swap })\left(A_{2}^{\prime} \otimes B_{2}^{\prime}\right) \\
= & \left(A_{1}^{\prime} U_{B}^{\prime} A_{2}^{\prime}\right) \otimes\left(A_{2}^{\prime} U_{A}^{\prime} A_{1}^{\prime}\right) .
\end{aligned}
$$


Theorem 4. The necessary conditions that $n$ applications of a $U_{d}\left(c_{1}, c_{2}, c_{3}\right)$-class gate can simulate any two-qubit unitary operations are

$$
\begin{aligned}
n\left(c_{1}+c_{2}-\left|c_{3}\right|\right) & \geq \frac{3 \pi}{4}, \\
n\left(c_{1}-c_{2}-\left|c_{3}\right|+\frac{\pi}{4}\right) & \geq \frac{3 \pi}{4} .
\end{aligned}
$$

Proof. Since we have supposed that $n$ applications of the $U_{d}\left(c_{1}, c_{2}, c_{3}\right)$-class gate can simulate any two-qubit gate, for continuity, it should be able to simulate the vertex points of the geometric representation of non-local two-qubit unitary operation, i.e. it should be able to simulate the vertex point $U_{d}\left(\frac{\pi}{4}, \frac{\pi}{4}, \frac{\pi}{4}\right)$ and $U_{d}\left(\frac{\pi}{4}, \frac{\pi}{4},-\frac{\pi}{4}\right)$ (Though they are the same gate, but their neighbor points in the tetrahedral representation are different from each other.). So we can obtain

$$
\begin{aligned}
& n \phi\left(U_{d}\left(c_{1}, c_{2}, c_{3}\right)\right) \succ \phi\left(U_{d}\left(\frac{\pi}{4}, \frac{\pi}{4}, \frac{\pi}{4}\right)\right), \\
& n \phi\left(U_{d}\left(c_{1}, c_{2}, c_{3}\right)\right) \succ \phi\left(U_{d}\left(\frac{\pi}{4}, \frac{\pi}{4},-\frac{\pi}{4}\right)\right) .
\end{aligned}
$$

There must be

$$
n\left(c_{1}+c_{2}-\left|c_{3}\right|\right) \geq \frac{3 \pi}{4} .
$$

On the other hand, using lemma 3 , we can get that if $n$ applications of $U_{d}\left(c_{1}, c_{2}, c_{3}\right)$ can simulate any two-qubit gate,

$$
\overline{U_{d}}\left(c_{1}, c_{2}, c_{3}\right)=U_{d}\left(\frac{\pi}{4}-\left|c_{3}\right|, \frac{\pi}{4}-c_{2}, \operatorname{sign}\left(c_{3}\right)\left(c_{1}-\frac{\pi}{4}\right)\right)
$$

can also construct any two-qubit unitary operation with $n$ applications. This is because that $U_{d}\left(\frac{\pi}{4}-\left|c_{3}\right|, \frac{\pi}{4}-c_{2}, \operatorname{sign}\left(c_{3}\right)\left(c_{1}-\frac{\pi}{4}\right)\right)$ is equivalent to $U_{d}\left(c_{1}, c_{2}, c_{3}\right) U_{d}\left(\frac{\pi}{4}, \frac{\pi}{4},-\operatorname{sign}\left(c_{3}\right) \frac{\pi}{4}\right)$, and two consecutive $U_{d}\left(\frac{\pi}{4}, \frac{\pi}{4},-\operatorname{sign}\left(c_{3}\right) \frac{\pi}{4}\right)$ are equivalent to local unitary operations (If $n$ is an odd number, we can multiply a SWAPclass gate on the gate to be simulated. It does not affect our proof since the gate to be simulated is an arbitrary two-qubit gate). Note here sign function is defined as

$$
\begin{aligned}
& \operatorname{sign}(x)=1, \text { when } x \geq 0, \\
& \operatorname{sign}(x)=-1, \text { when } x<0 .
\end{aligned}
$$

From this discussion, it can be obtained that

$$
\begin{aligned}
& n \phi\left(\overline{U_{d}}\left(c_{1}, c_{2}, c_{3}\right)\right) \succ \phi\left(U_{d}\left(\frac{\pi}{4}, \frac{\pi}{4}, \frac{\pi}{4}\right)\right), \\
& n \phi\left(\overline{U_{d}}\left(c_{1}, c_{2}, c_{3}\right)\right) \succ \phi\left(U_{d}\left(\frac{\pi}{4}, \frac{\pi}{4},-\frac{\pi}{4}\right)\right) .
\end{aligned}
$$

So we can get that

$$
n\left(c_{1}-c_{2}-\left|c_{3}\right|+\frac{\pi}{4}\right) \geq \frac{3 \pi}{4} .
$$

In the situation that $c_{2}=c_{3}=0$, i.e. the elementary gate is a controlled- $\mathrm{U}$ gate, it can be obtained that the minimum applications required to implement any arbitrary two-qubit gate together with local gates is $\left\lceil\frac{3 \pi}{4 c_{1}}\right\rceil(\lceil x\rceil$ is the minimum integer number that is not smaller than $x$.). It is just the same condition proposed by J. Zhang et al. [8], and it was also proved to be sufficient there.

Theorem 5. B gate is the unique gate (up to local unitary operations) that can simulate any two-qubit gate with only two applications.

Proof. Here the B gate is just the $U_{d}\left(\frac{\pi}{4}, \frac{\pi}{8}, 0\right)$ gate which was proposed by J. Zhang et al. [7]. According to Theorem 4 , if two applications of $U_{d}\left(c_{1}, c_{2}, c_{3}\right)$ can simulate any two-qubit gate, three parameters of the gate must satisfy

$$
\begin{aligned}
& c_{1}+c_{2}-\left|c_{3}\right| \geq \frac{3 \pi}{8}, \\
& c_{1}-c_{2}-\left|c_{3}\right| \geq \frac{\pi}{8} .
\end{aligned}
$$


Since $\frac{\pi}{4} \geq c_{1} \geq c_{2} \geq\left|c_{3}\right|$, there must be

$$
c_{1}=\frac{\pi}{4}, c_{2}=\frac{\pi}{8}, c_{3}=0 .
$$

We can understand that $c_{3}$ must be zero in another point of view. For continuity, two applications of this gate should be able to construct the identity gate, i.e. $U_{d}\left(c_{1}, c_{2}, c_{3}\right)$ should be equivalent to $U_{d}^{-1}\left(c_{1}, c_{2}, c_{3}\right)$. Comparing the Makhlin's invariants [14,15] of $U_{d}\left(c_{1}, c_{2}, c_{3}\right)$ and $U_{d}^{-1}\left(c_{1}, c_{2}, c_{3}\right)$, we can obtain that $c_{3}=0$. The sufficiency of this theorem has been proved by J. Zhang et al. [7].

Applying theorem 4 in the case of $n=3$, we can obtain the following corollary directly.

Corollary 6 . The necessary conditions that a gate $U_{d}\left(c_{1}, c_{2}, c_{3}\right)$ can simulate any arbitrary two-qubit unitary operation with three applications are

$$
\begin{aligned}
& c_{1}+c_{2}-\left|c_{3}\right| \geq \frac{\pi}{4}, \\
& c_{1}-c_{2}-\left|c_{3}\right| \geq 0 .
\end{aligned}
$$

As mentioned above, the CNOT, Double CNOT (DCNOT) and Super controlled gates [17] all can construct any two-qubit gate with three applications. They all satisfy this condition. However, one half of perfect entanglers [11] which can generate maximal entanglement states from product states can not construct an arbitrary two-qubit gate with only 3 applications. This result was shown in Fig. 1. (For convenience, we only depict the tetrahedron $O A C F$ : $\frac{\pi}{4} \geq c_{1} \geq c_{2} \geq c_{3} \geq 0$. It is not difficult to depict the tetrahedron $\frac{\pi}{4} \geq c_{1} \geq c_{2} \geq-c_{3} \geq 0$ by symmetry.). It is somewhat a surprising result, since that all perfect entanglers have the same entangling ability as CNOT without auxiliary system. For example, $\sqrt{S W A P}$ gate which is represented by $U_{d}\left(\frac{\pi}{8}, \frac{\pi}{8}, \frac{\pi}{8}\right)$ does not satisfy the condition but it is a perfect entangler. $\sqrt{S W A P}$ can be produced by exchange interaction Hamiltonian $J \overrightarrow{S_{1}} \cdot \overrightarrow{S_{2}}$, which is typical in solid systems. In addition, it is the most powerful gate in this class, but at least 6 applications of it is required to implement an arbitrary two-qubit unitary operation.

\section{Figure 1.}

We conjecture that

$$
P=\min \left\{c_{1}+c_{2}-\left|c_{3}\right|, \frac{\pi}{4}+c_{1}-c_{2}-\left|c_{3}\right|\right\}
$$

can measure the ability of a gate to construct a universal two-qubit unitary operation. For this measurement, the B gate is the most powerful one, the SWAP gate and local unitary gate have the smallest ability. In general, at least $\left\lceil\frac{3 \pi}{4 P}\right\rceil$ applications is needed for a $U_{d}\left(c_{1}, c_{2}, c_{3}\right)$-class gate to construct an arbitrary two-qubit gate.

An arbitrary two-qubit gate can also be constructed with different types of non-local gates. For example, three parameter-tunable $(S w a p)^{\alpha}$ gate can simulate an arbitrary two-qubit gate [16] but three parameter-fixed $(S w a p)^{\alpha}$ gate can not. Hence, it was also proved that 3 applications of a nontrivial Hamiltonian can simulate any two-qubit unitary operations [15]. However, it is more difficult to solve the optimality for different fixed gates acting as elementary gates in general. Though we can introduce the necessary conditions by the same way as we have mentioned above, it is not easy to get an exact form. But in some simple cases such as controlled-U gate, we can get the following result.

Theorem 7. [17] If a two-qubit gate $U_{d}\left(c_{1}, c_{2}, 0\right)$ can be simulated by $U_{d}\left(\gamma_{1}, 0,0\right)$ and $U_{d}\left(\gamma_{2}, 0,0\right)$, the parameters must satisfy the following conditions.

$$
\begin{aligned}
\gamma_{1}+\gamma_{2} & \geq c_{1}+c_{2}, \\
\left|\gamma_{1}-\gamma_{2}\right| & \leq c_{1}-c_{2} .
\end{aligned}
$$

Proof: Since that

$$
\phi\left(U_{d}\left(\gamma_{1}, 0,0\right) U_{d}\left(\gamma_{2}, 0,0\right)\right) \succ \phi\left(U_{d}\left(c_{1}, c_{2}, 0\right)\right),
$$

it can be obtained that $\gamma_{1}+\gamma_{2} \geq c_{1}+c_{2}$.

On the other hand, if $U_{d}\left(\gamma_{1}, 0,0\right)$ and $U_{d}\left(\gamma_{2}, 0,0\right)$ can simulate $U_{d}\left(c_{1}, c_{2}, 0\right), U_{d}\left(c_{1}, c_{2}, 0\right)$ and $U_{d}^{-1}\left(\gamma_{2}, 0,0\right)$ which is equivalent to $U_{d}\left(\gamma_{2}, 0,0\right)$ also can simulate $U_{d}\left(\gamma_{1}, 0,0\right)$. Using the lemma 3 , it can be obtained that

$$
\phi\left(U_{d}\left(c_{1}, c_{2}, 0\right) \overline{U_{d}}\left(\gamma_{2}, 0,0\right)\right) \succ \phi\left(U_{d}\left(\gamma_{1}, 0,0\right)\right),
$$


that is $c_{1}-c_{2} \geq \gamma_{1}-\gamma_{2}$. Similarly, $c_{1}-c_{2} \geq \gamma_{2}-\gamma_{1}$. So there should be $\left|\gamma_{1}-\gamma_{2}\right| \leq c_{1}-c_{2}$.

Note J. Zhang et al. [8] have proved that two controlled-U gate can not construct a $U_{d}\left(c_{1}, c_{2}, c_{3}\right)$ gate with $c_{3} \neq 0$.

It is known that realizing quantum computation needs to decompose the desired unitary operation into elementary gates. Earlier work to complete this decomposition is to find the universal two-qubit gate, and this needs infinite time use of a fixed two-qubit elementary gate in almost all of the case. Another more practical way is to use a fixed non-trivial two-qubit gate (excluding SWAP and identity gates) together with arbitrary one-qubit gates. This method can construct an arbitrary unitary operation with finite time using of elementary gates. On the other hand, the realization of some "standard" gate, e.g. CNOT, is not always efficient by different Hamiltonian. Investigation of the optimal construction with general two-qubit entangling gates is required. The necessary condition for optimal construction of a two-qubit unitary operation is proposed in this paper. It can be proved that this condition is also sufficient in the case of two applications of the elementary two-qubit gate, i.e. the B gate is the unique gate that can construct a two-qubit universal circuit with only two applications. It is also shown that one half of perfect entanglers can not simulate any two-qubit gate with only 3 applications. However, there are many open questions of the optimal simulation, e.g. the sufficient conditions of Theorem 4, i.e. the optimal operable construction is desired.

Simulating a gate by another gate is also different from Hamiltonian simulation which allows infinitely many steps of evolution $[3,4,18,19]$. The gate simulation, i.e. finite times of switch on the Hamiltonian is more practical in realization of an actual quantum process.

This work was supported by the National Fundamental Research Program (2001CB309300), the National Natural Science Foundation of China (No. 10304017), the Innovation Funds from Chinese Academy of Sciences (CAS).

[1] D. Deutsch et al., Proc. R. Soc. Lond. A 449, 669 (1995); D. P. DiVincenzo, Phys. Rev. A 51, 1015 (1995); T. Sleator and H. Weinfurter, Phys. Rev. Lett. 74, 4087 (1995); A. Barenco, Proc. R. Soc. Lond. A 449, 678 (1995); S. Lloyd, Phys. Rev. Lett. 75, 346 (1995).

[2] J. L. Dodd, M. A. Nielsen, M. J. Bremner, and R. T. Thew, Phys. Rev. A 65, 040301 (2002); W. Dür, G. Vidal, J. I. Cirac, N. Linden, and S. Popescu, Phys. Rev. Lett. 87, 137901 (2001); M. A. Nielsen, M. J. Bremner, J. L. Dodd, A. M. Childs, and C. M. Dawson, Phys. Rev. A 66, 062317 (2002); P. Wocjan, D. Janzing, and T. Beth, Quantum Inf. Comput. 2, 117 (2002).

[3] C. H. Bennett et al., Phys. Rev. A 66, 012305 (2002).

[4] G. Vidal and J. I. Cirac, Phys. Rev. A 66, 022315 (2002).

[5] A. Barenco et al., Phys. Rev. A 52, 3457 (1995).

[6] G. Vidal and C. M. Dawson, Phys. Rev. A 69, 010301 (2004); F. Vatan and C. Williams, Phys. Rev. A 69, 032315 (2004); V. V. Shende, I. L. Markov, and S. S. Bullock, Phys. Rev. A 69, 062321 (2004).

[7] J. Zhang, J. Vala, S. Sastry, and K. B. Whaley, Phys. Rev. Lett. 93, 020502 (2004).

[8] J. Zhang, J. Vala, S. Sastry, and K. B. Whaley, Phys. Rev. A 69, 042309 (2004).

[9] M. J. Bremner et al., Phys. Rev. Lett. 89, 247902 (2002).

[10] N. Khaneja, R. Brockett, and S. J. Glaser, Phys. Rev. A 63, 032308 (2001).

[11] B. Kraus and J. I. Cirac, Phys. Rev. A 63, 062309 (2001).

[12] K. Fan, Proc. Natl. Acad. Sci. U.S.A. 35, 131 (1949).

[13] A. M. Childs, H. L. Haselgrove, and M. A. Nielsen, Phys. Rev. A 68, 052311 (2003).

[14] Y. Makhlin, Quant. Inf. Proc. 1, 243 (2002); quant-ph/0002045.

[15] J. Zhang, J. Vala, S. Sastry, and K. B. Whaley, Phys. Rev. A 67, 042313 (2003).

[16] H. Fan, V. Roychowdhury, and T. Szkopek, quant-ph/0410001.

[17] M.-Y. Ye, Y.-S. Zhang, and G.-C. Guo, quant-ph/0407108 v1.

[18] G. Vidal, K. Hammerer, and J. I. Cirac, Phys. Rev. Lett. 88, 237902 (2002); H. L. Haselgrove, M. A. Nielsen, and T. J. Osborne, Phys. Rev. A 68, 042303 (2003); M. J. Bremner, D. Bacon, and M. A. Nielsen, quant-ph/0405115.

[19] R. Zeier, M. Grassl, and T. Beth, Phys. Rev. A 70, 032319 (2004). Figure caption.

All gates represented by points in tetrahedron $A B C D$ can simulate any two-qubit unitary operations with only 3 applications. Every point in tetrahedron $A B C D, B C D E$ or $A B D E$ represents a perfect entangler. Here the coordinates of points $O, A, B, C, D, E$ and $F$ are $(0,0,0),\left(\frac{\pi}{4}, 0,0\right),\left(\frac{\pi}{4}, \frac{\pi}{8}, 0\right),\left(\frac{\pi}{4}, \frac{\pi}{4}, 0\right),\left(\frac{\pi}{8}, \frac{\pi}{8}, 0\right),\left(\frac{\pi}{8}, \frac{\pi}{8}, \frac{\pi}{8}\right)$ and $\left(\frac{\pi}{4}, \frac{\pi}{4}, \frac{\pi}{4}\right)$ respectively. 


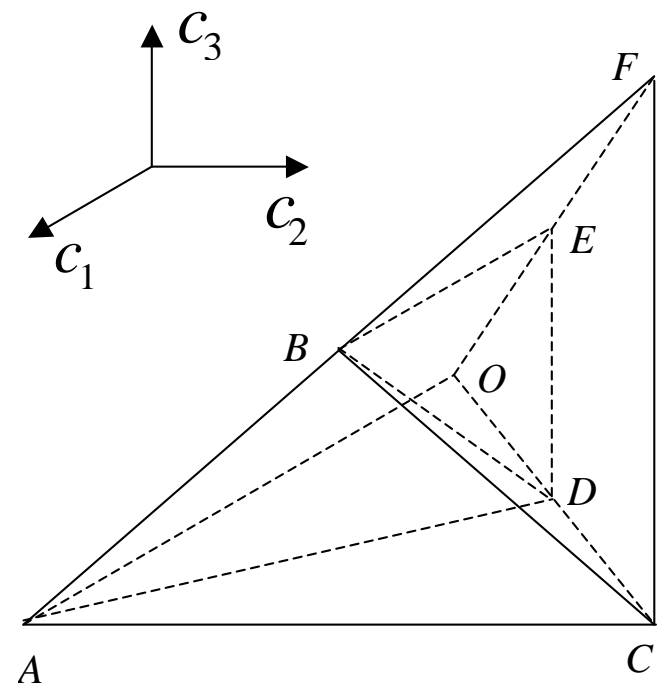

Figure 1 